\title{
Impact of Body Site, Age, and Gender on the Collagen/Elastin Index by Noninvasive in vivo Vertical Two-Photon Microscopy
}

\author{
Carolin Czekalla $^{a}$ Karl-Heinz Schönborn ${ }^{b}$ Nadine Döge ${ }^{a}$ Sora Jung ${ }^{a}$ \\ Maxim E. Darvin ${ }^{a} \quad J u ̈ r g e n ~ L a d e m a n n^{a}$ Martina C. Meinke ${ }^{a}$ \\ ${ }^{a}$ Department of Dermatology, Venereology, and Allergology, Charité - Universitätsmedizin Berlin, and \\ ${ }^{b}$ WOM World of Medicine GmbH, Berlin, Germany
}

\section{Keywords}

Second harmonic to autofluorescence aging index of dermis - Wide-field 2-photon microscopy . Autofluorescence $\cdot$ Second harmonic generation $\cdot$ Skin aging

\section{Abstract}

Background/Aims: Extrinsic and intrinsic skin aging is subject to constant remodeling and degradation processes, primarily in components of the extracellular matrix. While collagen fibers thin out during the aging process, the amorphous elastin fibers accumulate. These are essential formative components of the dermis. So far, these processes have been detected in vertical histological sections of invasive biopsies and recently in noninvasive horizontal scans. Methods: In this pilot study, a modified noninvasive 2-photon microscope was applied to measure the collagen/elastin index of skin in vivo. The obtained images permit an immediate vertical survey and allow a conclusion on the dermal composition at once. The collagen/elastin index was quantified by the second harmonic to autofluorescence aging index of dermis (SAAID) depending on volunteers' age (18-66 years), gender, and body area. Results: The highest SAAID was measured at the volar forearm as compared to the ab-

\section{KARGER}

๑๑ 2017 S. Karger AG, Basel

E-Mail karger@karger.com

www.karger.com/spp dominal SAAID, which was significantly lower $(p<0.05)$. The gluteal region showed the significantly lowest SAAID $(p<$ 0.05 ). The SAAID in female skin was higher compared to male skin and decreased with increasing age. Conclusion: These effects are to be considered in subsequent studies to be able to specifically detect and evaluate influences.

(c) 2017 S. Karger AG, Basel

\section{Introduction}

Skin aging is influenced by intrinsic, e.g., genetic aspects, and extrinsic, e.g., environmental factors, such as sun radiation, toxins, and pollutants [1]. Two main parameters of the extracellular matrix (ECM), which are required to determine the extent of skin aging, are the structure proteins collagen (70-80\%) for strength and elastin $(2-4 \%)$ for elasticity of the skin. They are concentrated in the stratum papillare [2].

Various studies have shown that the collagen/elastin ratio as well as the collagen and elastin structures change with age [3-6]. The elasticity of the skin increases with atrophy of the collagen fiber bundles, the related flattening of the rete ridges, and the thickening of the dermal elastic fibers. There is an accumulation of amorphous ag-

Prof. Dr. Martina C. Meinke

Charité - Universitätsmedizin Berlin

Klinik für Dermatologie, Venerologie und Allergologie, Charitéplatz 1

DE-10117 Berlin (Germany)

E-Mail martina.meinke@ charite.de 
glutinated material and a distinctly thinned-out elastin net. In case of extrinsic skin aging, these structural changes can also be observed [7]. Especially the accumulation of elastin in the dermis represents the degree of extrinsic skin aging $[8,9]$. Solar radiation is the most important environmental factor causing skin aging [10-13]. Exposure to solar radiation induces free radicals, which interact with the ECM determinates once a critical threshold is reached [14]. This effect is called photoaging or premature skin aging $[1,8,15]$.

Currently the slicing of skin biopsies is the gold standard method to obtain a vertical histological thin section of the skin for diagnosis $[3,16]$. The invasive extraction of skin biopsies is limited to a certain extent and area due to ethical reasons. To overcome these obstacles, in this study a noninvasive technique based on 2-photon microscopy (2PM) is applied. A modification of the currently used 2PM allows to image vertical skin sections in histomorphological correlation down to a certain depth sufficient to image the dermal structure of the skin. The flexibility provided by the $x, y$, and $z$ axes enables horizontal scans ex vivo as well as vertical scans ex vivo and in vivo. In contrast to other scanning microscopic methods, for instance, laser scanning microscopy (LSM) and other multiphoton microscopy types, this $2 \mathrm{PM}$ equipped with so-called flying optics has an extended field of view of up to $7 \mathrm{~mm}$ in $x$ direction (lateral) and $500 \mu \mathrm{m}$ in $z$ direction (vertical) [17]. This provides a quicker, larger, and more comprehensive view into the skin as well as an impression of the ECM state [18]. Optical coherence tomography, as another noninvasive technique, enables to image the skin in the same orientation [8]; however, contrary to $2 \mathrm{PM}$ or LSM, the currently available in vivo devices are unsuitable for the detection of cellular structures or ECM components due to the limited resolution of 10-20 $\mu \mathrm{m}$. Recently, LSM and 2PM have been applied in vivo to analyze and quantify the collagen and elastin structures in the skin noninvasively $[4,7,19-22]$.

The signal collection of the LSM does not allow the distinction between collagen and elastin signals [22, 23], which is important for a detailed analysis. The wavelength-dependent collection of the emitted signal provided by $2 \mathrm{PM}$ enables a strict differentiation between the signals; the autofluorescence (AF) signal and the second harmonic generation (SHG) signal are detected [24-26]. The SHG signal is only induced in ordered structures such as collagen I, which is mainly responsible for the elasticity and decreases with age [27]. The AF signal is emitted by elastin fibers under $2 \mathrm{PM}$ excitation from 740 to $780 \mathrm{~nm}$, the maximum emission wavelength ranging around 480 $\mathrm{nm}$. The AF reflects NAD(P)H, FAD, and other skin fluorophores within the cytoplasm of skin cells, keratin, melanin in pigment cells and in the basal layer $[14,20,25$, 28].

The SHG-to-AF aging index of dermis (SAAID) is a noninvasive, objective parameter for determining the intrinsic and extrinsic aging on various skin areas [23]. The collagen/elastin index will be quantified with the SAAID, which is defined as:

$$
S A A I D=(S H G-A F) /(S H G+A F) .
$$

SAAID scoring is a powerful method for analyzing the dermis dependent on AF and SHG signal intensities [29]. The index indicates the fibrotic status of the dermis. The SAAID decreases with photoaging depending on an enhanced AF signal intensity from increased elastosis and a reduced SHG signal originating from a decreasing amount of collagen fibers [14, 16, 21, 24, 30-32].

The purpose of the present study was to evaluate alterations in the dermal ECM components. Differences and effects of body area, gender and age of skin on the SAAID were statistically analyzed in images of noninvasive wide-field $2 \mathrm{PM}$.

First results about the determination of the SAAID and its dependence on the body area have already been published in a letter [33].

\section{Material and Methods}

Subjects

Ten healthy female and 10 male volunteers from Germany of skin type II, according to the Fitzpatrick classification, were included in the study [34]. The subjects were divided into 3 groups of age: age group 1 from 18 to 30 years $(n=7)$, age group 2 from 31 to 50 years $(n=7)$, and age group 3 from 50 to 66 years $(n=6)$. Pregnant women and nursing mothers, subjects accommodated in an institution via judicial or an administrative order (as per $\$ 29$ of the German Medical Devices Act) or persons with known drug addiction or alcohol abuse were excluded from the study. The study was approved by the ethics committee of Berlin (DIMDI No.: 00008670 (A), Eudamed No.: CIV-13-08-0115553). Prior informed written consent was obtained from all volunteers.

Two-Photon Microscopy

The system used in this study to implement the innovative 2PM FlySCAN technology was a specific in vivo 2PM VertiSCAN V.21 (WOM World of Medicine GmbH, Berlin, Germany) which enables to increase the maximal lateral dimension of the skin area imaged by 2PM. Here, a recently developed lateral scan movement called "flying optics" for 2PM was implemented. The setup of the device, measuring procedure, and parameters of the study have already been described in detail [18]. Briefly, the lateral scan is achieved by moving the focus together with the ultra-lightweight

Noninvasive 2PM to Measure Effects on

Skin Pharmacol Physiol 2017;30:260-267 

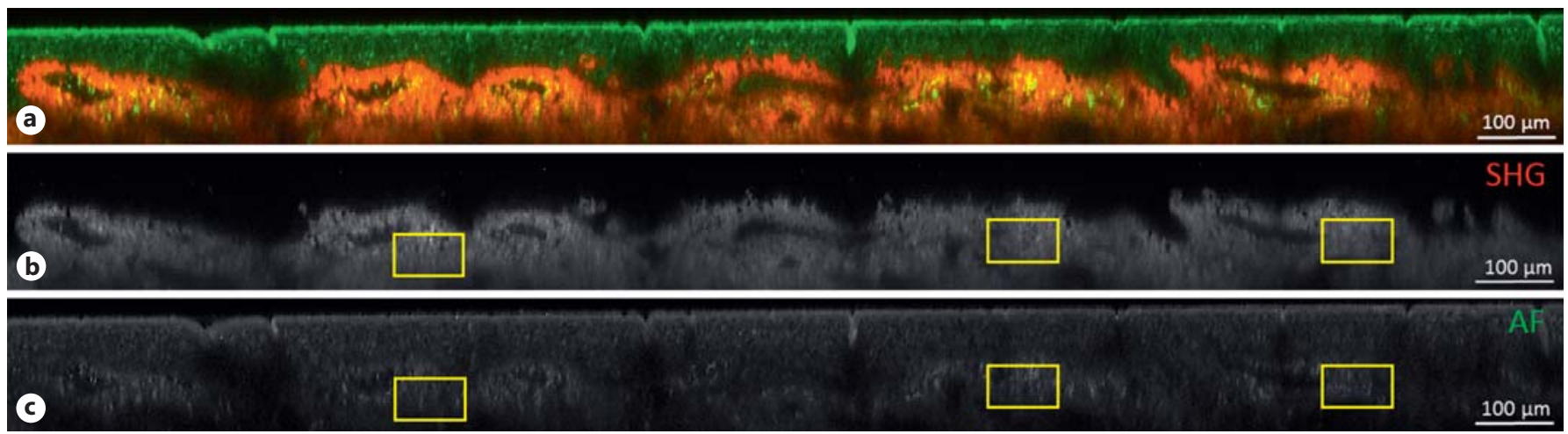

Fig. 1. In vivo vertical AF and SHG skin images of a shaved, tape-stripped skin specimen of the abdomen of a 37-year-old woman. a False-color image. b, c Gray images showing SHG (b) and AF signals (c) for exemplary areas of even size (yellow marked areas). The calculated SAAID objectified the image (a) that permits a subjective assessment of skin aging. $2,000 \times 180 \mu \mathrm{m}$.

(14 g) high-aperture (numeric aperture $=1.25$ ) scan lens. The galvanometer scanner of the "classical" $2 \mathrm{PM}$ is replaced by a highdefinition $(0.1 \mu \mathrm{m})$ and high-scan speed (up to $40 \mathrm{~mm} / \mathrm{s}$ ) linear piezo-driven scan motor. AF and SHG images are generated simultaneously after an excitation at $780 \mathrm{~nm}$. The emitted signals were detected between 380 and $410 \mathrm{~nm}$ (channel 1) as SHG signal and in the range between 415 and $720 \mathrm{~nm}$ (channel 2) as AF signal [17]. The optical resolution of pixel size between 0.5 and up to 50 $\mu \mathrm{m}^{2}$ can be randomly selected [18]. In this way a change in magnification as in classical light microscopy can be used to find the area of interest.

\section{Procedure}

Five areas in different skin sites were examined including volar and dorsal forearm, abdomen (hip bone region), gluteal region (sacral), and sural region. These skin sites were selected due to their variety of sun exposure, different elastic strain, and accessibility. Due to the good accessibility, the forearm is frequently investigated for standardized measurements in both in vivo and ex vivo studies $[4,7]$. In a previous study, ECMs of the skin in the gluteal and abdominal regions were investigated invasively by taking biopsies [2,3]. With the 2PM VertiSCAN V.21 it was possible to image skin areas of the abdominal, gluteal, and sural regions noninvasively in vivo. Prior to the $2 \mathrm{PM}$ measurements, the thickness of the stratum corneum was reduced by tape stripping (tesa ${ }^{\circledR}$-Film, Art. No.: 5529, Beiersdorf, Hamburg, Germany). This procedure was evaluated in a former study [18]. The tapes were consecutively stripped 5 times from each measuring site of $2 \times 4 \mathrm{~cm}$ in order to reduce the scattering on the stratum corneum. The 5 skin sites were acquired from each volunteer. One overview scan (axis: 2,000 $\times 400 \mu \mathrm{m}$, image resolution: $10 \mu \mathrm{m}^{2}$, speed: $10 \mathrm{~mm} / \mathrm{s}$ ) was followed by a detailed scan in the area of interest with a resolution of $1 \mu \mathrm{m}^{2}$. To call up the image, the processing software DataViewer Version 1.3 was applied at an image resolution of 1 pixel $=1 \mu \mathrm{m}$.

\section{Determination of the SAAID}

Three square areas of approximately $4,400 \mu \mathrm{m}^{2}$ each were analyzed per scan regarding the AF and SHG signal intensity. The marked areas were located within the upper dermis excluding ar- eas with enhanced blood circulation or decreased signal intensity which is shown in Figure 1. On average the center of the area was located about $100 \mu \mathrm{m}$ below the surface. The square areas were labeled and analyzed in exactly the same position in both AF and SHG images, and the obtained gray values were analyzed using ImageJ 1.41 (Java 1.6.0_45). The SAAID was determined from the calculated mean of AF and SHG values according to equation 1. It has to be considered that the SAAID provides arbitrary numeric values which depend on the specific device. The values are evaluable within the conduced study, but not quantitatively comparable with the results obtained with other measuring devices in previous studies.

\section{Statistics}

Microsoft Excel 2010 and SPSS 22 (IBM ${ }^{\circledR}$ SPSS $^{\circledR}$ Statistics) for Windows 2010 were used for statistical analyses. Data were analyzed by nonparametric, paired, and unpaired tests including, if appropriate, analysis of (co-)variance, Kruskal-Wallis test, MannWhitney and Wilcoxon tests. The level of significance was assumed at $p<0.05$.

\section{Results}

A combined in vivo false-color image (Fig. 1a) and the 2 gray value images of SHG (Fig. 1b) and AF (Fig. 1c) of an $x-z$ scan of the 2PM are shown in Figure 1. The AF signal is depicted in green, whereas the SHG signal is shown in red. The cellular structure of the epidermis displayed in green can be clearly differentiated from the red-colored collagen fibers. Because of the high resolution and differential signal collection, collagen I could be quantified in the images. Due to the clear transition of the dermoepidermal junction zone, the basal lamina can be visualized. The AF signal of the overlying pigmented basal cells of the 


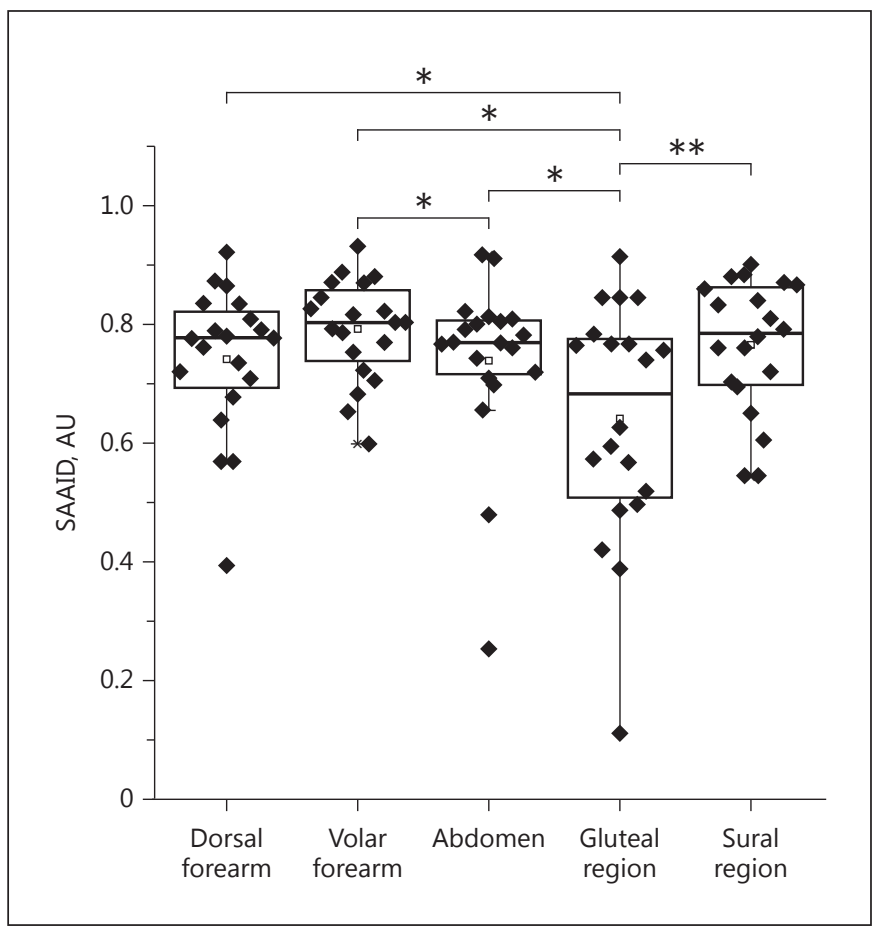

Fig. 2. Boxplot of SAAID of the 5 body areas of all volunteers. The boxplots show medians as horizontal bars and the mean values as little squares. Significant values are determined as $* p<0.05$ and ${ }^{* *} p<0.01$ (Wilcoxon test). The whiskers show maximal 1.5 times of the interquartile range.
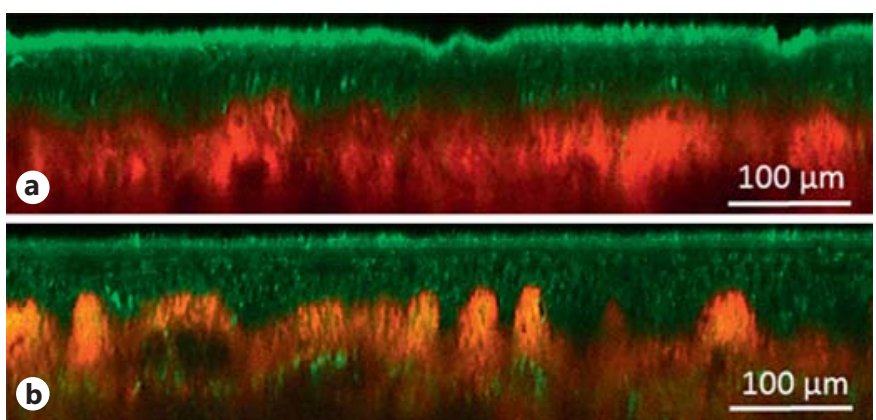

Fig. 3. Vertical 2PM skin image. a, b In vivo false-color pictures of a shaved, tape-stripped female skin specimen of the sural region: comparison of young (a) and aged (b) skin. a 25-year-old skin shows a mainly dense network of collagen in the dermis. b 65-yearold skin. Collagen fibers are scattered and less numerous in $\mathbf{b}$ than in a. $680 \times 160 \mu \mathrm{m}$.

basal lamina is more intense compared to the other epidermal cells except the keratinocytes. The AF signal of the elastin fibers in the dermis is overlain by the much higher SHG signal; thus, the elastin fibers in the dermis appear in yellow. Blood vessels in the papillary layer were identi-

Noninvasive 2PM to Measure Effects on the Collagen/Elastin Index fiable due to the structural characteristic loops appearing in black without AF or SHG signals.

\section{Differences in Skin Areas}

The SAAID depends on the elastin and collagen structures which are distinct at various skin sites. Figure 2 presents the boxplot of the SAAID for different skin sites. Generally, the SAAID is highest at the volar forearm and decreases from the sural region to the dorsal forearm and abdomen. The lowest SAAID is found in the gluteal region, although this area is well protected from solar radiation. The SAAID is significantly reduced on the abdomen compared to the volar forearm $(p=0.023$, Wilcoxon test). In addition, the SAAID of the gluteal region is significantly lower compared to all other body areas $(p<$ 0.05 , Wilcoxon test). There is a tendency towards a higher SAAID at the leading forearm compared to the dorsal forearm.

\section{Influences of Age}

Collagen fibers are visible as vertical fibers (red colored) in the stratum papillare in Figures 1a and 3. A higher AF signal in aged skin indicates a greater presence of elastin aggregates, which is shown in Figure 3b. In Figure $3 \mathrm{~b}$ the dermal fibers changed color to orange or yellow, because the red-colored SHG signal decreases. Figure 4 gives an overview of the SAAID of different body areas within each defined age group and in comparison to the other age groups. Generally, the SAAID decreases with increasing age. This decrease is significant for the dorsal forearm ( $p=0.017$, Kruskal-Wallis test) and gluteal region ( $p=0.02$, Kruskal-Wallis test). Also the decrease in the SAAID from age group 1 to 2 on the dorsal forearm and from age group 1 to 3 in the gluteal region is significant $(p<0.01$, Mann-Whitney test).

\section{Effect of Gender}

The index of female subjects is always higher regardless of the measured body area. This applies to all 5 body areas as shown in Figure 5. The SAAID in female skin is significantly higher compared to that in male skin in the sural region ( $p=0.004$, Mann-Whitney test).

\section{Discussion}

The results of the presented study confirmed the impact of age on the ECM. With increasing age the collagen/ elastin index determined by the SAAID decreased. The mean SAAID values of age group 1 were significantly

Skin Pharmacol Physiol 2017;30:260-267 
Fig. 4. Boxplot of SAAID comparing the same body areas with different ages. ${ }^{* *} p<$ 0.01 . The SAAID of the dorsal forearm of age group 1 is significantly higher compared to that of age group 2 at $p=0.009$ (Mann-Whitney test). Also, the SAAID of the gluteal region of age group 1 is significantly above that of age group 3 at $p=0.007$ (Mann-Whitney test). The boxplots show medians as horizontal bars and the mean values as little squares. The whiskers show maximal 1.5 times of the interquartile range.

Fig. 5. Boxplot of SAAID values comparing the measured skin sites of male and female subjects. The boxplots show medians as horizontal bars and the mean values as little squares. ${ }^{* *} p<0.01$ (Mann-Whitney test). The whiskers show maximal 1.5 times of the interquartile range.
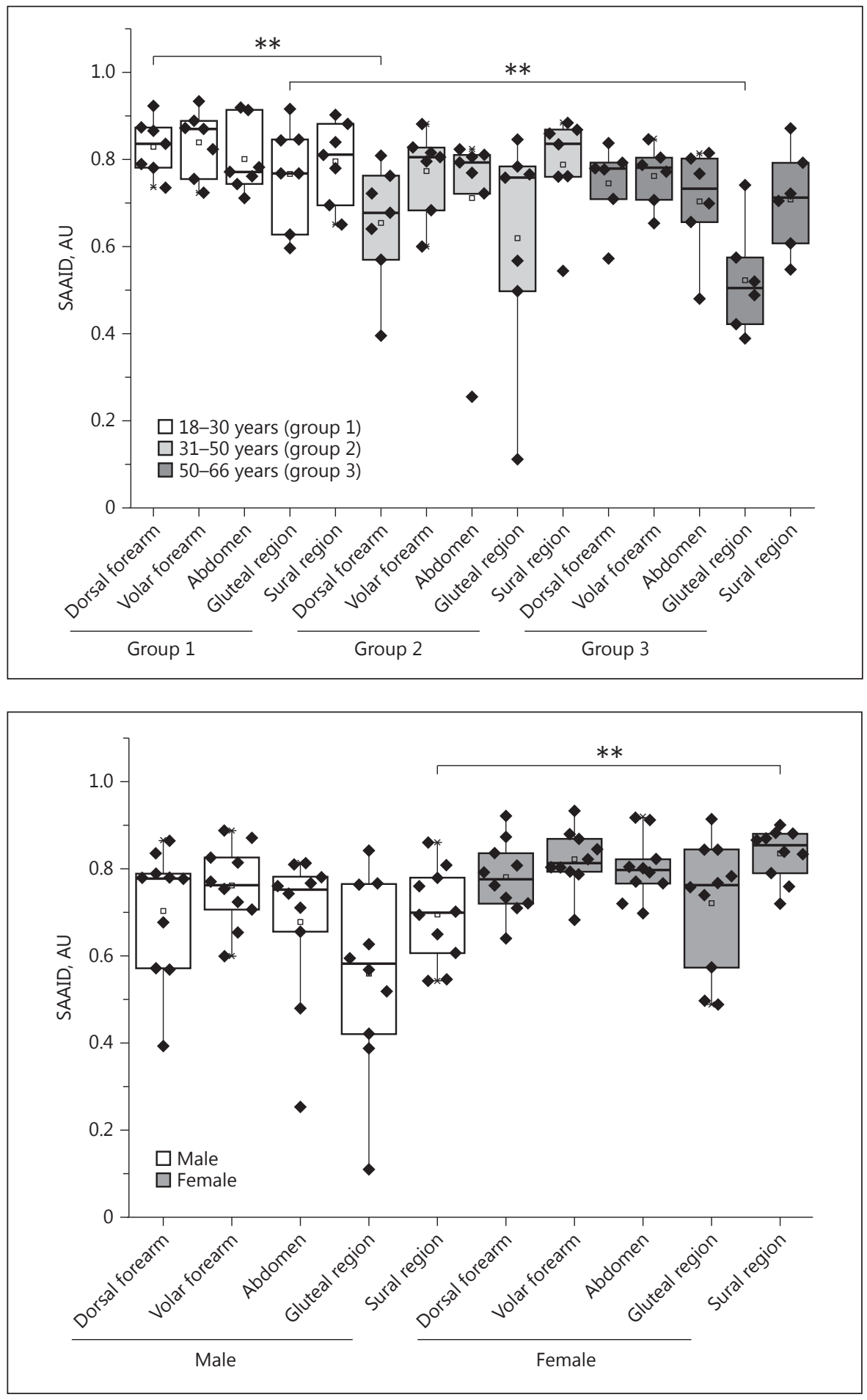

higher compared to the ones of all other age groups, but no significant differences between age group 2 and 3 were found. Koehler et al. [7] used morphological changes determined by multiphoton microscopy from aged skin called dermis morphology score and found the same sig- nificant decrease with age. Baillie et al. [8] have shown that influences of age could strongly correlate with photoaging.

In our pilot study, an individually varying and cumulated solar exposure in the respective groups could ex- 
plain the differences between them. Furthermore, other intrinsic and environmental impacts, such as the use of a sunscreen or smoking have to be considered in future studies. The present study was not intended to investigate the influence of short-term dermal solar exposure as the investigations had been carried out between the end of September and December in Berlin (Germany) to avoid individual influences of recent solar radiation on the SAAID. Nevertheless, minor differences between the dorsal and volar forearm SAAID were observed. Lower SAAID values on the dorsal forearm suggest a physiologically lower elasticity compared to the volar forearm [8].

Sanchez et al. [4] could show that sun-exposed skin generally had a lower SAAID than sun-protected skin. A similar effect was observed in a study which compared the influence of 4 weeks of solar irradiation on sunscreentreated and untreated skin [14].

In this context, the analyses conducted by Lin et al. [19] on excised skin are interesting showing an alteration of collagen and elastin induced by heating. SHG and AF images were analyzed with the AF-to-SHG index of dermis (ASID $=\mathrm{AF} / \mathrm{SHG})$, which increased when exposed to $60^{\circ} \mathrm{C}$ for more than $20 \mathrm{~min}$.

The significant decrease in elastosis material in sundamaged skin was identified by histology by Karagas et al. [6] and Baillie et al. [8], who found a significant increase in the prevalence of solar elastosis $(p \leq 0.001)$ with age.

Interestingly, in our own study, we found a low SAAID value in the sun-protected gluteal region. In this body area a high collagen/elastin ratio and therefore a high SAAID is assumed. These results show that noninvasive methods provide a suitable and important tool to determine the SAAID of the different body areas, which is important to avoid ethical problems.

So far, the collagen/elastin ratio was investigated in several independent studies on different body areas (forearm $[4,5,7,14]$, face (cheek) [9], not defined [16, 29]). Whether or not the results obtained using different methods in various studies are comparable is questionable. The present study provides a direct comparison of the SAAID from different body areas in vivo as a single both reproducible and noninvasive method was applied.

The investigation of the different body areas in the presented study clearly indicates that not only the solar exposure is responsible for the SAAID value. As discussed above, the sun-exposed dorsal forearm should have the lowest SAAID value, but interestingly the abdomen and sural region have similar SAAID values, whereas the gluteal region exhibits significantly lower values. Only the

Noninvasive 2PM to Measure Effects on the Collagen/Elastin Index sun-protected volar forearm shows higher values. Mechanical influences and movements could also cause a higher elastic strain as expected on the strongly expanded gluteal skin area. It is necessary that comparable skin sites must be selected when comparing photo-aged to sunprotected skin areas.

The data could be complemented by investigating further skin areas (e.g., the face and the upper back), but these areas were not accessible by the used 2PM. However, this system is one of the very few microscopes permitting noninvasive vertical scans of the skin at such a high resolution that even cells in the epidermis can be differentiated. Furthermore, it allows imaging the epidermis and dermis in one image in a certain plane of one skin area, which makes the determination of the SAAID highly reproducible.

In addition to the influence of the skin area, in this pilot study, the influence of the gender on the SAAID was investigated. In the literature, different results were reported regarding the effect of the gender on the collagen/ elastin ratio. In 1 study, Koehler et al. [35] demonstrated that older females exhibit a faster, steeper SAAID decrease with age than males, which could not be confirmed by subsequent investigations $[5,7]$. Our results indicate that females have higher SAAID values than males with age, although the mean age of the female group was higher compared to the male group. Still, the low number of investigated subjects is to be considered when interpreting the results.

\section{Conclusion}

This pilot study confirmed the influence of age on the ECM as already shown in the literature. Furthermore, a first indication was found that the SAAID might be influenced by gender, and it was possible to establish significant differences between different photo-protected body areas as they were measured with the same method within one study.

Future studies will have to take into account also other factors influencing the parameter under investigation to avoid any undesired effects.

\section{Acknowledgments}

We would like to thank the World of Medicine GmbH for providing the 2PM, and the Foundation of Skin Physiology for financial support. 


\section{Statement of Ethics}

The study was approved by the ethics committee of Berlin (DIMDI No.: 00008670 (A), Eudamed No.: CIV-13-08-0115553). Prior informed written consent was obtained from all volunteers.

\section{Author Contributions}

C.C., N.D. and S.J. performed research, K.-H.S. contributed the 2PM device, J.L., M.C.M., and M.E.D. designed the research study, C.C. and S.J. analyzed the data, C.C. and M.C.M. wrote the paper.

\section{Disclosure Statement}

The authors state no conflict of interest.

\section{References}

1 Manickavasagam A, Hirvonen LM, Melita LN, Chong EZ, Cook RJ, Bozecc L, Festy F: Multimodal optical characterisation of collagen photodegradation by femtosecond infrared laser ablation. Analyst 2014;139:61356143.

2 Lovell C, Smolenski K, Duance V, Light N, Young S, Dyson M: Type I and III collagen content and fibre distribution in normal human skin during ageing. Br J Dermatol 1987; 117:419-428.

3 Knott A, Reuschlein K, Lucius R, Stab F, Wenck H, Gallinat S: Deregulation of versican and elastin binding protein in solar elastosis. Biogerontology 2009;10:181-190.

4 Sanchez WY, Obispo C, Ryan E, Grice JE, Roberts MS: Changes in the redox state and endogenous fluorescence of in vivo human skin due to intrinsic and photo-aging, measured by multiphoton tomography with fluorescence lifetime imaging. J Biomed Opt 2013; 18:061217.

5 Koehler MJ, Preller A, Kindler N, Elsner P, Konig K, Buckle R, Kaatz M: Intrinsic, solar and sunbed-induced skin aging measured in vivo by multiphoton laser tomography and biophysical methods. Skin Res Technol 2009; 15:357-363.

6 Karagas MR, Zens MS, Nelson HH, Mabuchi K, Perry AE, Stukel TA, Mott LA, Andrew AS, Applebaum KM, Linet M: Measures of cumulative exposure from a standardized sun exposure history questionnaire: a comparison with histologic assessment of solar skin damage. Am J Epidemiol 2007;165:719-726.

7 Koehler MJ, Hahn S, Preller A, Elsner P, Ziemer M, Bauer A, Konig K, Buckle R, Fluhr JW, Kaatz M: Morphological skin ageing criteria by multiphoton laser scanning tomography: non-invasive in vivo scoring of the dermal fibre network. Exp Dermatol 2008;17: 519-523.

8 Baillie L, Askew D, Douglas N, Soyer HP. Strategies for assessing the degree of photodamage to skin: a systematic review of the literature. Br J Dermatol 2011;165:735-742.

9 Sugata K, Osanai O, Sano T, Takema Y: Evaluation of photoaging in facial skin by multiphoton laser scanning microscopy. Skin Res Technol 2011;17:1-3.
10 Krutmann J: How the sun ages our skin. The dermis as the driving force (in German). Hautarzt 2011;62:588-590.

11 Biniek K, Levi K, Dauskardt RH: Solar UV radiation reduces the barrier function of human skin. Proc Natl Acad Sci USA 2012;109: 17111-17116.

12 Robert C, Bonnet M, Marques S, Numa M, Doucet O: Low to moderate doses of infrared A irradiation impair extracellular matrix homeostasis of the skin and contribute to skin photodamage. Skin Pharmacol Physiol 2015; 28:196-204.

13 Akhalaya MY, Maksimov GV, Rubin AB, Lademann J, Darvin ME: Molecular action mechanisms of solar infrared radiation and heat on human skin. Ageing Res Rev 2014;16: $1-11$.

14 Darvin ME, Richter H, Ahlberg S, Haag SF, Meinke MC, Le Quintrec D, Doucet O, Lademann J: Influence of sun exposure on the cutaneous collagen/elastin fibers and carotenoids: negative effects can be reduced by application of sunscreen. J Biophoton 2014;7: 735-743.

15 Proksch E, Schunck M, Zague V, Segger D, Degwert J, Oesser S: Oral intake of specific bioactive collagen peptides reduces skin wrinkles and increases dermal matrix synthesis. Skin Pharmacol Physiol 2014;27:113-119.

16 Cicchi R, Sestini S, De Giorgi V, Massi D, Lotti T, Pavone FS: Nonlinear laser imaging of skin lesions. J Biophoton 2008;1:62-73.

17 Döge N, Thiel E, Seewald G, Albrecht S, Vogt A, Liebscher J, Schönborn K-H: Wide-field two photon microscopy. Opt Photon 2015;10: 39-42.

18 Czekalla C, Schönborn KH, Markworth S, Ulrich M, Göppner D, Gollnick H, Röwert-Huber J, Darvin ME, Lademann J, Meinke MC: Technical parameters of vertical in vivo multiphoton microscopy: a critical evaluation of the flyscanning method. Laser Phys Lett 2015; 12:085602.

19 Lin MG, Yang TL, Chiang CT, Kao HC, Lee JN, Lo W, Jee SH, Chen YF, Dong CY, Lin SJ: Evaluation of dermal thermal damage by multiphoton autofluorescence and secondharmonic-generation microscopy. J Biomed Opt 2006;11:064006.
20 Toki F, Honkura N, Shirakata Y, Imamura T, Higashiyama S, Nanba D: Second harmonic generation reveals collagen fibril remodeling in fibroblast-populated collagen gels. Cell Struct Funct 2013;38:227-236.

21 Perry SW, Burke RM, Brown EB: Two-photon and second harmonic microscopy in clinical and translational cancer research. Ann Biomed Eng 2012;40:277-291.

22 Darvin ME, Richter H, Zhu YJ, Meinke MC, Knorr F, Gonchukov SA, Koenig K, Lademann J: Comparison of in vivo and ex vivo laser scanning microscopy and multiphoton tomography application for human and porcine skin imaging. Quantum Elec 2014;44: 646-651.

23 Sugata K, Osanai O, Sano T, Akiyama M, Fujimoto N, Tajima S, Takema Y: Evaluation of unique elastic aggregates (elastic globes) in normal facial skin by multiphoton laser scanning tomography. Eur J Dermatol 2015;25: 138-144.

24 König K, Speicher M, Kohler MJ, Scharenberg R, Kaatz M: Clinical application of multiphoton tomography in combination with high-frequency ultrasound for evaluation of skin diseases. J Biophoton 2010;3: 759-773.

25 Schenke-Layland K, Riemann I, Damour O, Stock UA, Konig K: Two-photon microscopes and in vivo multiphoton tomographs - powerful diagnostic tools for tissue engineering and drug delivery. Adv Drug Deliv Rev 2006;58:878-896.

26 Konig K: Clinical multiphoton tomography. J Biophoton 2008;1:13-23.

27 Waller JM, Maibach HI: Age and skin structure and function, a quantitative approach. II. Protein, glycosaminoglycan, water, and lipid content and structure. Skin Res Technol 2006; 12:145-154.

28 Zipfel WR, Williams RM, Christie R, Nikitin AY, Hyman BT, Webb WW: Live tissue intrinsic emission microscopy using multiphoton-excited native fluorescence and second harmonic generation. Proc Natl Acad Sci USA 2003;100:7075-7080. 
29 Cicchi R, Kapsokalyvas D, De Giorgi V, Maio V, Van Wiechen A, Massi D, Lotti T, Pavone FS: Scoring of collagen organization in healthy and diseased human dermis by multiphoton microscopy. J Biophoton 2010;3:3443.

30 Adur J, Carvalho HF, Cesar CL, Casco VH: Nonlinear optical microscopy signal processing strategies in cancer. Cancer Inform 2014; 13:67-76.
31 Roberts MS, Dancik Y, Prow TW, Thorling CA, Lin LL, Grice JE, Robertson TA, Konig K, Becker W: Non-invasive imaging of skin physiology and percutaneous penetration using fluorescence spectral and lifetime imaging with multiphoton and confocal microscopy. Eur J Pharm Biopharm 2011;77:469-488.

32 Del Barco O, Bueno JM: Second harmonic generation signal in collagen fibers: role of polarization, numerical aperture, and wavelength. J Biomed Opt 2012;17:045005.

33 Czekalla C, Schönborn KH, Döge N, Jung S, Darvin ME, Lademann J, Meinke MC: Body regions have an impact on the collagen/elastin index of the skin measured by non-invasive in vivo vertical two photon microscopy. Exp Dermatol 2017, Epub ahead of print.
34 Fitzpatrick TB: The validity and practicality of sun-reactive skin types I through VI. Arch Dermatol 1988;124:869-871.

35 Koehler MJ, Konig K, Elsner P, Buckle R, Kaatz M: In vivo assessment of human skin aging by multiphoton laser scanning tomography. Opt Lett 2006;31:2879-2881. 